Cita bibliográfica: García Gónzalez, E.D., López Guevara, V.M.y López Pardo,G. (2022). Análisis de la resiliencia social en sistemas socio-ecológicos: una propuesta interdisciplinaria para los destinos turísticos y su desarrollo sostenible. Investigaciones Turísticas (23), pp. 48-72. https://doi.org/10.14198/INTURI2022.23.3

\title{
Análisis de la resiliencia social en sistemas socio-ecológicos: una propuesta interdisciplinaria para los destinos turísticos y su desarrollo sostenible.
}

\author{
Analysis of social resilience in socio-ecological systems: an interdisciplinary proposal for the \\ sustainable development of tourism destinations
}

Erick David García González (iD, Universidad Nacional Autónoma de México, México errickgargon89@gmail.com

Víctor Manuel López Guevara iD, El Colegio de Tlaxcala A.C, México victorlopez@coltlax.edu.mx

Gustavo López Pardo (iD, Universidad Nacional Autónoma de México, México Ipardo@unam.mx

\section{RESUMEN}

En la búsqueda por avanzar hacia la sostenibilidad, se han abordado diversos paradigmas, entre los cuales, figura el de la resiliencia como atributo de los sistemas socio-ecológicos. La literatura científica da cuenta de diversos esfuerzos por modelar la resiliencia y su operacionalización, pero dando prioridad a los aspectos ecológicos sobre los sociales. En cuanto al turismo, se reconoce la necesidad por desarrollar aproximaciones que permitan ensayar su aplicación, pero sin faltar al reconocimiento de su naturaleza social y su dinámica compleja con el entorno. En este sentido, el presente escrito tiene por objetivo presentar una propuesta teórico-conceptual para el estudio de la resiliencia social en destinos turísticos, construida a partir del seguimiento de cuatro fases para la teorización, planteadas por Swedberg (2012). A partir de un análisis crítico de la literatura, se muestra la necesidad de indagar, desde una convergencia interdisciplinar, en cinco elementos para comprender la resiliencia social en un destino turístico: agencia local, capital social, gobernanza, espacio temporalidad y glocalidad. Esto permitió generar un modelo que enfatiza en las especificidades de la esfera social sin faltar a su inherencia ecológica, a la vez que expone al turismo como un fenómeno social inmerso en la dinámica de los socio-ecosistemas a multi escala. Así, las capacidades adaptativas y de transformación de los grupos sociales se evidencian como cualidades necesarias para enfrentar las repercusiones de sus impactos y poder transitar hacia un desarrollo sostenible en los destinos turísticos.

Palabras clave: Sistemas socio-ecológicos; resiliencia social; turismo; teorización. 


\section{ABSTRACT}

In the quest to move towards sustainability, several paradigms have been addressed, including resilience as an attribute of social-ecological systems. The scientific literature refers to different efforts to model resilience and its operationalisation, but prioritizing ecological aspects over social ones. With respect to tourism, there is a need to develop approaches to test its application, but without failing to recognise its social nature and its complex interactions with the environment. In this sense, the aim of this paper is to present a theoretical-conceptual proposal for the study of social resilience in tourism destinations based on the four phases for theorising proposed by Swedberg (2012). A document analysis reveals the need for an interdisciplinary approach to study five elements in order to understand social resilience in the tourist destination, local agency, social capital, governance, time-space and glocality. On this basis, a model has been developed to emphasise the specific characteristics of the social sphere, distinguishing it from the ecological one, while not losing sight of its inherent nature. It also exposes tourism as a social phenomenon immersed in the dynamics of multi-scale socio-ecosystems. Thus, the adaptive and transformative capacities of social groups are shown to be necessary qualities to cope with the repercussions of their impacts to achieve the sustainable development in tourism destinations.

Keywords: Socio-ecological systems; social resilience; tourism; theorizing

\section{INTRODUCCIÓN}

Derivado de la separación y distinción de disciplinas naturales y sociales por la evolución en la lógica y semántica epistemológica a partir del siglo XIX, el concepto de "resiliencia" ha sido utilizado para referir cualidades similares asociadas a diferentes objetos de estudio, lo que ha generado ambigüedad en la definición del concepto y en la operativización para su entendimiento (Puig y Rubio, 2011).

A partir de la segunda mitad del siglo pasado, el pensamiento académico intentó integrar distintas visiones (tanto de ciencias naturales, como sociales) para indagar en la comprensión de un mismo ente estudiado, en aras de avanzar en el conocimiento de la dinámica entre distintos elementos y los factores que pueden desencadenar cambios positivos o negativos. Ante ello, surgió la propuesta del estudio de los fenómenos desde la complejidad (Wagner, 1999; Gershenson, 2013) y el desarrollo de la teoría general de sistemas, la cual postula que cualquier conjunto de elementos interrelacionados (sin importar si éstos son de origen social o biológico) pueden ser visualizados como sistema mediante su abstracción gráfica, lo que facilita su análisis a partir del desarrollo de una explicación holística (Von Bertalanffy, 1969, 1973). Desde la disciplina de la ecología, esto fomentó un abordaje multidisciplinario alrededor de la resiliencia al conectarla con el tratamiento de los sistemas socio-ecológicos (Folke, 2006). No obstante, dentro de este encuadre se percibe un acotado análisis del ámbito social, lo cual limita la comprensión de las causas que explican por qué un objeto de estudio socio-ecológico tiende o no hacia la adaptación o transformación en una determinada escala (Walker et al., 2004; Biggs et al., 2012). En este orden de ideas, existen fenómenos cuya esencia se basa en las dinámicas sociales y la movilidad de las personas, lo cual genera diversas perturbaciones socio-ecológicas in situ que deben ser consideradas para una mejor comprensión y gestión de un contexto particular, tal como ocurre en con el caso de los destinos turísticos (Farrell y Twining-Ward, 2004), ello significa que para poder indagar en las dinámicas socio-ecológicas del turismo, es necesario generar un marco teórico que permita 
ahondar en los componentes a considerar para el estudio de su resiliencia, dentro de lo cual se propone marcar un énfasis en el ámbito social para reorientar la tendencia reconocida en los estudios previos.

Durante la construcción de un trabajo de investigación, es común que el autor replique definiciones y conceptos para posteriormente adoptar modelos y métodos preestablecidos para confirmar y fortalecer la validez de una teoría. Sin embargo, desde la perspectiva de Swedberg (2012), este proceder ha pausado el avance del conocimiento y ha limitado el proceso creativo en la investigación. El mismo autor comenta que la teorización es un proceso necesario para fomentar el progreso del conocimiento. Ésta debe realizarse en la fase previa al trabajo de campo, con el objetivo de construir un sistema de ideas que, de forma tentativa, expliquen el objeto de estudio. Si bien el ejercicio de teorizar demanda valorar y eventualmente retomar ideas y conceptos de distintos autores, evita el uso de concepciones preestablecidas que explican la realidad desde contextos espacio-temporales que necesariamente no responden a las condiciones en las cuales se plantea construir el nuevo conocimiento.

Teorizar requiere el cumplimiento de una serie de pasos que logren explicar un fenómeno de manera heurística (Moustakas, 1990); en dicho tenor, la teorización consta de las cuatro fases siguientes (Swedberg, 2012):

1. Observar. Permite registrar y sistematizar información sobre el objeto de estudio para caracterizarle y asegurar su entendimiento.

2. Formular un concepto central. Implica denotar y dar nombre a una problemática reconocida mediante la caracterización del objeto de estudio. Los términos empleados para señalar la problemática pueden retomarse de investigaciones previas.

3. Dar cuerpo al concepto central mediante la estructuración del objeto de estudio de manera heurística.

4. Formular y modelar una teoría tentativa que explique el objeto de estudio. Este apartado debe hacer énfasis en la relación de los elementos que la constituyen.

A partir de los argumentos anteriores, el presente escrito tiene como fin teorizar el concepto de resiliencia social, así como identificar los componentes a considerar para su análisis en los sistemas socio-ecológicos donde se haga presente la actividad turística mediante la operativización de las fases propuestas por Swedberg (2012). Para ello, previamente se efectuó una revisión documental en plataformas y repositorios académicos (Google Scholar, Researchgate y Redalyc) donde se consultaron artículos de revistas científicas especializadas y capítulos de libros académicos publicados en idioma inglés y español, vinculados a la temática de la resiliencia. Tras la revisión, se hizo una selección de más de 80 documentos académicos y representativos sobre su estudio desde el enfoque ingenieril, psicológico, ecológico y socio-ecológico. Esto permitió construir una base de datos para discernir la resiliencia social desde distintas disciplinas, así como identificar los elementos que cada una considera relevantes para su análisis.

Dicha revisión permitió para la primera fase, la descripción de la evolución del concepto de resiliencia en un orden cronológico para abarcar su acepción desde el enfoque de los sistemas socio-ecológicos, lo que facilitó durante la segunda fase, la identificación de la resiliencia social como concepto central que presenta vacíos de investigación y áreas de 
oportunidad para su análisis en el turismo. Para la tercera etapa, se explican de manera crítica los documentos que han abordado el estudio de la resiliencia social desde disciplinas como la ecología, antropología, geografía, psicología y sociología, a fin de identificar elementos concordantes para su entendimiento y que justifiquen heurísticamente la importancia del estudio de la resiliencia social a partir de los efectos del turismo en un socio-ecosistema. En la última fase, se propone a partir de los elementos asiduamente identificados y los alcances teórico-prácticos de distintos estudios de caso, un modelo teórico en el cual se distinguen los cinco atributos para el análisis de la resiliencia social de los destinos turísticos con una perspectiva coherente con la interdisciplina; el mismo puede contribuir en una mejor comprensión de este fenómeno al: 1) reconocer su no linealidad y complejidad; 2) entender las realidades de los distintos actores de manera más profunda; 3) lograr identificar o incentivar capacidades de adaptabilidad o transformabilidad en el sistema; y 4) fomentar una toma de decisiones más certera ante shocks y perturbaciones ${ }^{1}$ (figura 1 ).

Figura 1. Operativización de las fases para teorizar ejecutadas en el proceso de investigación.
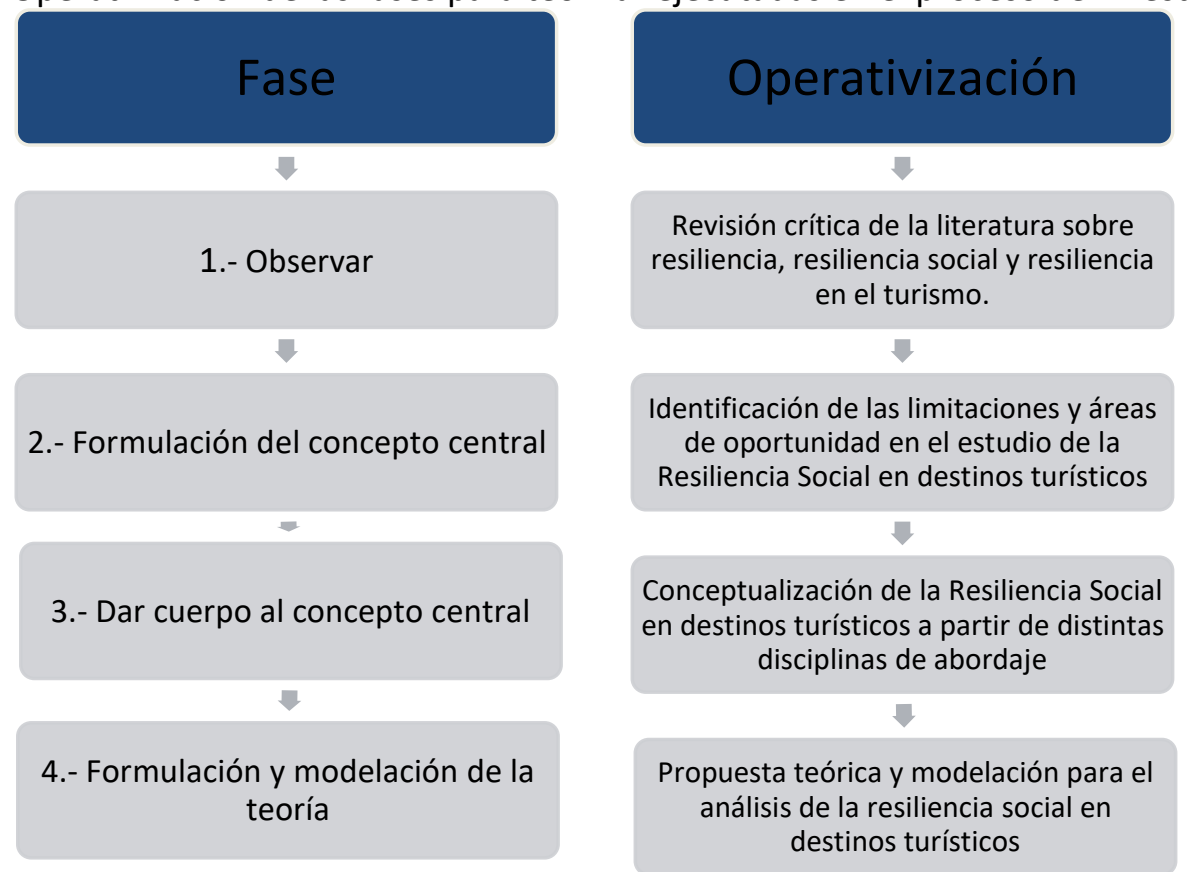

Fuente: elaboración propia con base en Swedberg, 2012.

En correspondencia con la estructura enunciada para este trabajo, en la siguiente sección se explica en qué consiste la resiliencia, al igual que su evolución como concepto en los sistemas socio-ecológicos.

\footnotetext{
${ }^{1}$ Entiéndase por shocks a los fenómenos que a corto plazo causan algún efecto masivo en el sistema, tales como pandemias huracanes, terremotos, atentados terroristas, etc. Y, por perturbaciones, aquellas situaciones que manifiestan su influencia en una escala determinada a mediano o largo plazo; estas pueden ser el monocultivo, el pastoreo, incorporación de políticas públicas, entre otras (Biggs et al., 2012; Keck y Sakdapolrak, 2013).
} 


\section{PRIMERA FASE: OBSERVACIÓN Y EXPLICACIÓN DEL FENÓMENO}

\subsection{Resiliencia: de una propiedad física a un paradigma multidisciplinario.}

La resiliencia implica, según su raíz etimológica (resilio), saltar o volver hacia atrás (Puig y Rubio. 2011). Fue a inicios del siglo XX cuando el término fue usado por primera vez en el ámbito de la ciencia moderna, específicamente en la física. En ese contexto, la resiliencia fue entendida como la propiedad que determina la capacidad de un cuerpo para regresar a su estado natural después de ser sometido a una deformación derivada de la aplicación de una fuerza externa (Fielding, 1937).

Entre las décadas de 1940 y 1950, diversos autores vinculados a la psiquiatría social emplearon el término para referir a la capacidad de niñas y niños para afrontar situaciones de estrés (Scoville, 1942; Bowlby, 1951, 1969; Atwool, 2006; Puig y Rubio, 2011). De esa forma, en el ámbito de la salud mental y la psicología, la resiliencia se asumió como una característica necesaria en los individuos para superar la adversidad (Rutter, 1971; 1985).

Hacia la década de 1970, en medio del advenimiento de los enfoques multidisciplinario y sistémico, el concepto en cuestión fue adoptado por el paradigma del sistema ecológico para referir a la "medida de la persistencia de los sistemas y su habilidad de absorber cambios y disturbios manteniendo las mismas relaciones entre poblaciones y las variables de estado" (Holling, 1973, p. 14).

A inicios del siglo XXI, la resiliencia cobró notoriedad al ser incorporada en la discusión teórico-conceptual vinculada a los sistemas socio-ecológicos, los cuales, según Berkes y Folke (2002) facilitan una aproximación holística que permite comprender la forma en la cual se presentan, adaptan y evolucionan las relaciones entre el entorno natural y las estructuras sociales. Al reconocer la compleja interacción entre personas y ecosistemas, Holling y Gunderson (2002) desarrollaron un modelo alusivo al ciclo adaptativo de los sistemas socioecológicos, al cual asociaron tres propiedades que propician el cambio del sistema. La primera de ellas es la potencialidad, a ella están asociados recursos, biomas y nutrientes acumulados, así como las distintas maneras en las cuales éstos pueden ser aprovechados; la segunda es la conectividad, la cual deriva de la interacción entre los elementos e instituciones incorporados en el sistema. Finalmente, la tercera propiedad es justamente, la resiliencia, misma que los autores definen como la capacidad del sistema socio-ecológico para gestionar los shocks y perturbaciones.

En el modelo de Holling y Gunderson (2002), la resiliencia se desarrolla a lo largo del bucle que dibuja, a través de cuatro fases, el tránsito del ciclo adaptativo (figura 2). En la transición que se identifica entre la fase $\boldsymbol{K}$ (conservación) y la $\boldsymbol{\Omega}$ (liberación), la resiliencia comienza a decrecer debido al exceso de conectividad y potencialidad de los elementos, esto genera mayor vulnerabilidad y puede conducir al colapso del sistema o bien a su transformación en función de sus características durante el tránsito entre la fase $\boldsymbol{\Omega}$ a la $\boldsymbol{\alpha}$ (reorganización). Es justo en esa fase del bucle donde el modelo propuesto reconoce el momento de mayor incertidumbre en el ciclo. Al superar ese tránsito, el sistema tiende a generar nuevas estrategias para gestionar los disturbios durante el transcurso de la fase $\boldsymbol{\alpha}$ a la $\boldsymbol{r}$ (explotación), con lo cual la resiliencia tiende a aumentar siempre y cuando se cuente con capacidades de adaptación y potencialidades pertinentes para preservar el funcionamiento adecuado del sistema ( $\boldsymbol{r}$ a $\boldsymbol{K}$ ) (Holling y Gunderson, 2002; Berkes y Folke, 2002). 
Figura 2. El ciclo adaptativo y resiliencia de los sistemas socio-ecológicos.

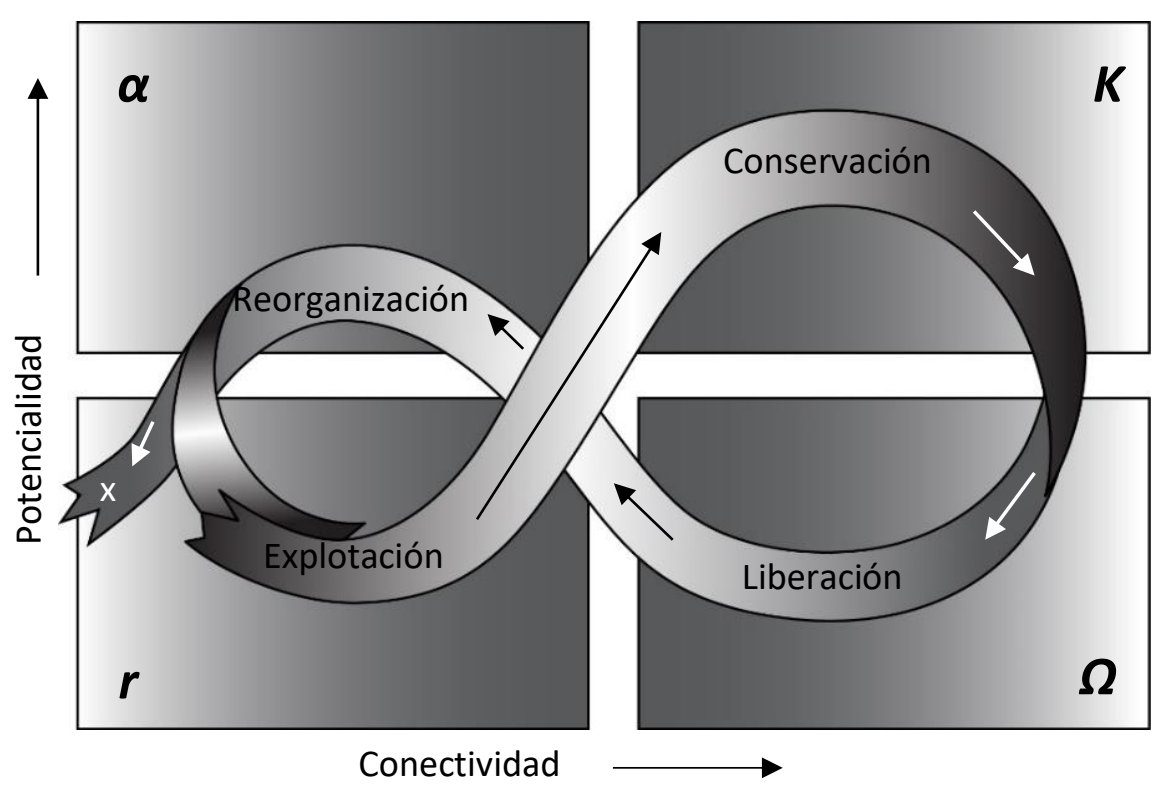

Fuente: Holling y Gunderson (2002).

Existen tres aspectos que permiten la resiliencia de los socio-ecosistemas (Walker et al., 2004; Zhu y Ruth, 2013; Hodgson et al., 2015):

- Latitud, que refiere al nivel máximo de cambio permitido por el sistema.

- Resistencia, indica la facilidad o dificultad que tiene un sistema para cambiar.

- Precariedad, el estado actual del sistema y qué tan cerca se encuentra del límite.

Todo sistema socio-ecológico, por su naturaleza compleja, se retroalimenta con otros al existir una interconexión entre ellos a macro y micro escala, proceso del cual emanan distintos shocks y perturbaciones (revuelta), mismos que pueden fomentar en los elementos del sistema a una escala determinada, la acumulación de experiencias y conocimientos que permitan dotarlos de capacidades para hacerles frente (rememoria) (Biggs et al., 2012). A esta interrelación se le conoce como panarquía (figura 3), y es necesaria su consideración para entender de mejor manera el curso de la resiliencia en el sistema que se desea analizar. 
Figura 3. La panarquía de los sistemas socio-ecológicos.

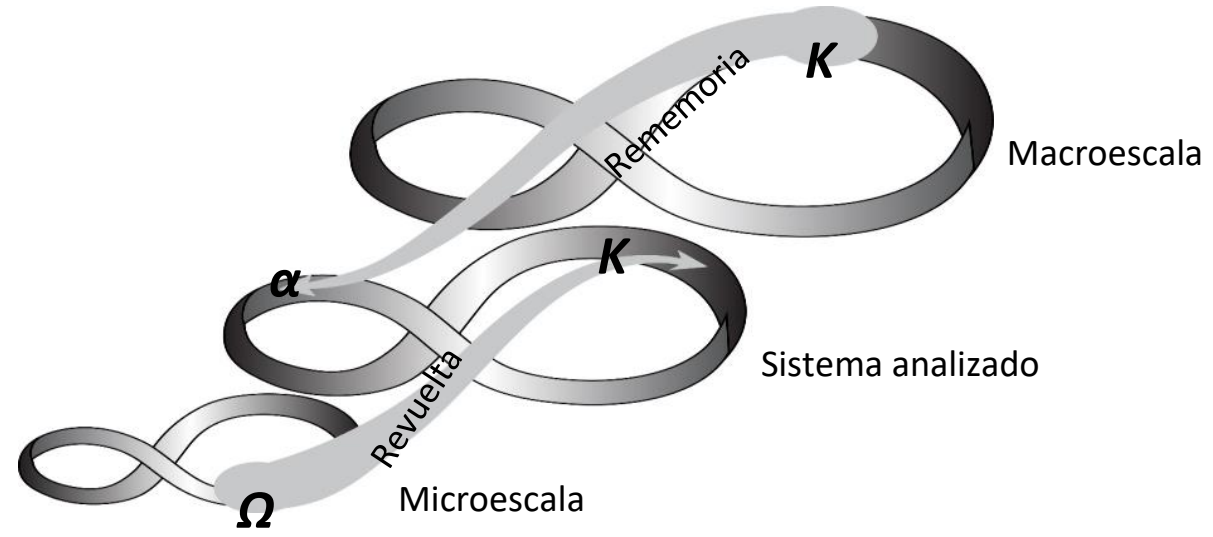

Fuente: Holling, Gunderson y Peterson, 2002; Folke (2006).

Así, derivado de las fluctuaciones ${ }^{2}$ que se presentan durante el ciclo adaptativo, pueden identificarse dos propiedades emergentes de la resiliencia (Walker et al., 2004; Folke, 2006).

- Adaptabilidad, capacidad de autoorganización del sistema.

- Transformabilidad, crear un nuevo sistema estable a partir de la introducción de nuevos componentes y nuevos modos de vida.

En ese sentido, la resiliencia puede interpretarse no solo como la capacidad de persistir y absorber los disturbios (Holling y Gunderson, 2002), sino también como la cualidad que tienen los sistemas socio-ecológicos (y sus elementos) para adaptarse al cambio e incluso de reinventarse (Folke, 2006); y desde las ciencias de la sostenibilidad, esto implica el uso de un paradigma interdisciplinario que adhiere nuevos elementos teórico-conceptuales al estudio y praxis del desarrollo sostenible, permitiendo la comprensión y resolución de las problemáticas complejas generadas entre socio-ecosistemas (Kates et al., 2001), lo cual puede ser de gran utilidad en contextos con presencia de actividad turística.

\subsection{La expresión de la resiliencia en el debate del turismo.}

La construcción del conocimiento turístico se ha desarrollado a través de dos pautas presentes desde la década de 1970. La primera de ellas, se ha caracterizado por la producción de saberes desde los enfoques uni y multidisciplinarios, principalmente de la economía, la geografía, la sociología y la psicología (Jafari y Ritchie, 1981; Jafari, 2005). La segunda, se ha desarrollado desde finales del siglo $X X$ en coherencia con el enfoque interdisciplinar (Theobald, 1998), y en respuesta a la necesidad de promover un abordaje holístico y sistémico capaz de contribuir a la gestión de los impactos generados por la actividad (Davidson, 2005).

En relación con la segunda pauta descrita, desde finales de los años 70 el turismo comenzó a ser ensayado desde la perspectiva de la Teoría General de Sistemas, con el objetivo de formular y poner a prueba modelos para explicar la dinámica compleja y multiescala del fenómeno turístico (Leiper, 1979; Mason, 2008; Tejeida et al., 2016).

\footnotetext{
${ }^{2}$ Entiéndase por fluctuaciones, a las variaciones que permiten identificar tendencias de los límites de cambio aceptable del sistema. Éstas dependerán del tipo de shock o perturbación, así como del (o los) elemento(s) que resulte(n) directamente afectado(s) (Folke, 2006; Biggs et al., 2012; Araral, 2013).
} 
A partir de la reformulación del modelo de Leiper (1979) y con base en la teoría de sistemas complejos, Jiménez (2005) ha perfilado un sistema turístico en el que existen diversas escalas de interacción. En todas ellas se presentan diversos mecanismos que inciden en la evolución del sistema, a saber:

- Entropía: nivel de transformación derivado de la interacción entre las regiones generadoras de turistas y del destino turístico.

- Neguentropía: información que tiende a estabilizar o disminuir el proceso entrópico del sistema.

- Homeóstasis: capacidad de estabilidad posterior al intercambio de información (entropía-negentropía).

- Retroalimentación: relación causa-efecto permanente que puede afectar al sistema de manera positiva o negativa.

Así, el modelo propuesto por Leiper pasa a ser sólo una parte del todo (el "subsistema turístico local"), ya que un destino turístico se interrelaciona con diversas regiones generadoras de turistas a la vez. Éste, simultáneamente forma parte de un "supersistema" de escala nacional y/o continental que en conjunto forman el "hipersistema" turístico. En ese sentido, los impactos económicos, sociales y ambientales, generados por el turismo son de carácter sinérgico y recursivo; por ende, lo que ocurra dentro de un contexto turístico local, afecta y es afectado por otro en diferentes escalas (figura 4).

Figura 4. Modelo del sistema turístico de Alfonso Jiménez.

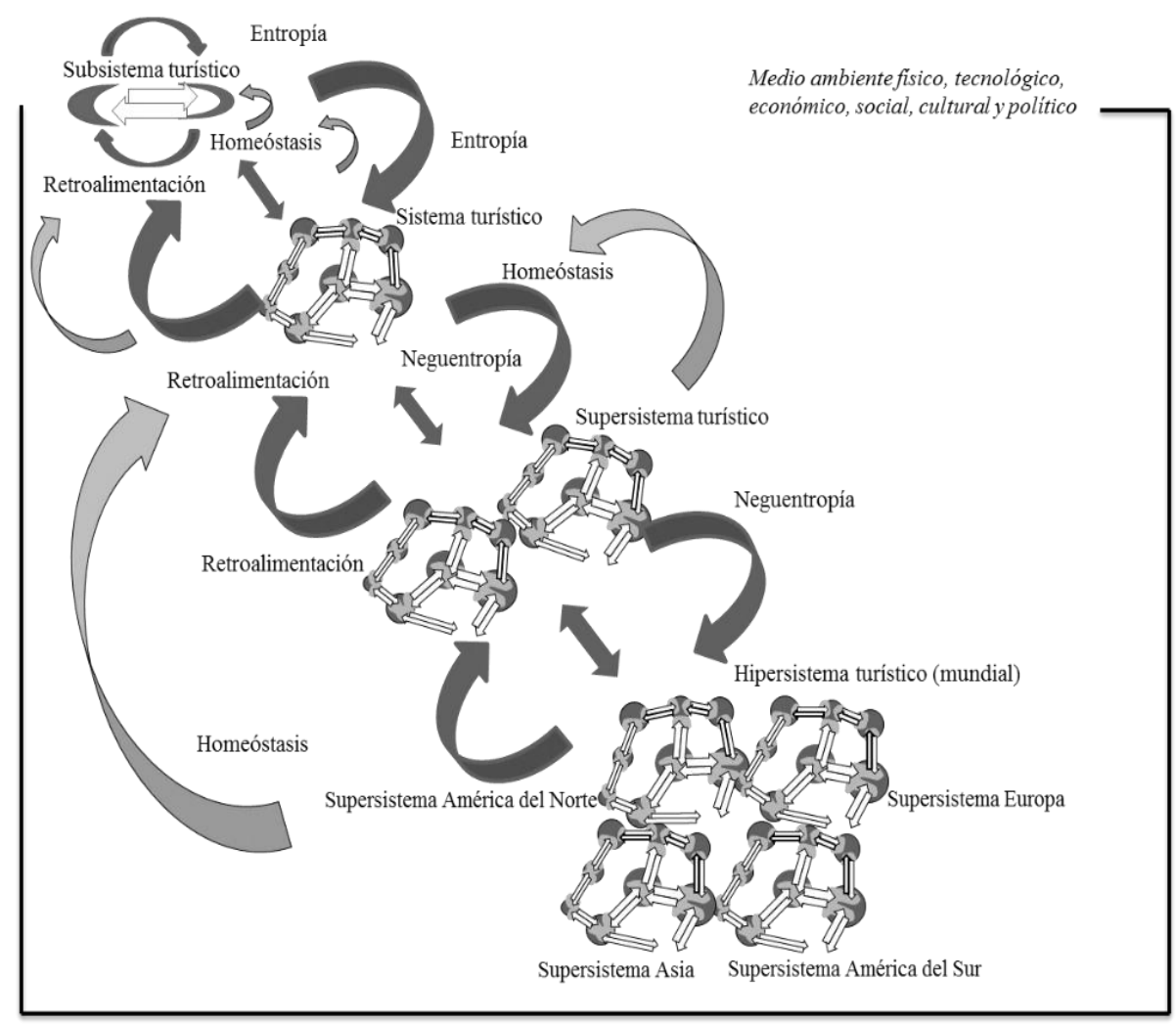

Fuente: Jiménez (2005). 
El modelo expuesto, denota la presencia del turismo de forma global. Sin embargo, le concibe -al igual que muchos otros modelos- como un sistema per se enfocado en el análisis exclusivo de los elementos que participan en la dinámica turística. En congruencia con el enfoque de los sistemas socio-ecológicos y la panarquía (Allen et al., 2014), resulta factible virar hacia una perspectiva holística y descentralizada del turismo para analizarlo como un fenómeno que puede causar diversos shocks y perturbaciones a multi escala socioecosistémica; reconceptualización que puede permitir una mirada distinta en la gestión de los destinos al avanzar en el reconocimiento de la complejidad turística, así como en el entendimiento de las capacidades de adaptación y transformación de los elementos que son alterados por el turismo de manera directa o indirecta (Farrel y Twining-Ward, 2004), sobre todo en el ámbito de los grupos de personas. Por tal motivo el siguiente apartado tiene la intención de remarcar por qué se debe avanzar en los vacíos de investigación vinculados con la resiliencia social para un mejor entendimiento de la complejidad turística.

\section{SEGUNDA FASE: FORMULACIÓN DEL CONCEPTO CENTRAL}

\subsection{Resiliencia Ecológica ¿y Social? del Turismo.}

Ostrom (2009) argumenta que se requieren mayores esfuerzos para indagar y comprender la compleja interconexión y retroalimentación entre socio-ecosistemas, por lo que es necesario analizarlos a partir de observaciones cualitativas y cuantitativas. Si bien la finalidad de la resiliencia y su modelación como parte del ciclo adaptativo, es ampliar la comprensión de las dinámicas complejas entre las personas y el ecosistema, los elementos e interacciones del componente social se incluyeron con base en modelos ecológicos que no han profundizado en su observación y evaluación, por lo que requieren mayor estudio al tener características propias cuyo análisis debe diferir del aplicado para los elementos biológicos, sobre todo en dinámicas que implican procesos de aprendizaje para toma de decisiones ante un shock o perturbación, o de gestión de los recursos disponibles en el sistema (Williams, B. and Hummelbrunner, 2010; Biggs et al., 2012).

En atención a los razonamientos anteriores, es necesaria mayor intervención de las ciencias sociales en el paradigma de la resiliencia (Folke, 2006), sobre todo al considerar que características como resistencia, latitud y precariedad, son de constructo netamente ecológico (Walker et al., 2004). Adicionalmente, las propiedades de adaptabilidad y transformabilidad, dependen en gran medida de las capacidades de los elementos sociales del sistema y la eficiencia con la que se gestionen los shocks y perturbaciones, por lo que operacionalizar la medición del ámbito social es primordial para avanzar en el análisis de la resiliencia de un contexto determinado (González-Quintero y Ávila-Foucat, 2019).

En ese sentido, si el sistema socio-ecológico se compone de dos esferas complejas (Adger, 2000; Berkes y Folke, 2002), es pertinente hacer la distinción entre una resiliencia ecológica y otra social, entendiendo por esta última - de forma preliminar - al ámbito del cual depende la expresión y el alcance de procesos de adaptación y transformabilidad que se presentan en una comunidad, bajo condiciones específicas, como respuesta ante la dinámica entre sistemas a multi escala. Esto resulta relevante para el estudio de los destinos turísticos, ya que eso puede delimitar de forma más clara las fluctuaciones causadas por este fenómeno, sobre todo en cuestión de las perturbaciones de origen antropogénico y que afectan al socioecosistema a largo plazo (Bec et al., 2015). Así, el siguiente apartado desarrolla las posturas variadas sobre resiliencia social, a fin de argumentar por qué profundizar en su análisis de 
manera holística, se muestra como una oportunidad de investigación en contextos donde la actividad turística se manifiesta.

\section{TERCERA FASE: ESTRUCTURACIÓN DEL CONCEPTO CENTRAL}

\subsection{La Resiliencia Social. Diversas posturas de abordaje}

Distintas disciplinas han tratado de contribuir en el entendimiento de la resiliencia social. Desde la geografía humana, Adger (2000) y Buckle (2006), sostienen que existe una serie de elementos e indicadores a contemplar en su análisis y que no han sido abordados en profundidad desde el enfoque de los sistemas socio-ecológicos, tales como el capital social, la organización comunitaria, así como la distribución y gestión del espacio geográfico. Respecto a este último, se enfatiza la necesidad de indagar en su manifestación mediante constructos como la seguridad alimentaria, variabilidad del mercado, migración e índice de empleo. Desde la perspectiva de esta disciplina, son elementos importantes debido a que las condiciones geográficas imponen para cada situación, distintas capacidades a desarrollar para los grupos humanos. Sin embargo, esto no debe significar la centralización del estudio de la resiliencia social ante shocks de origen natural (Paton y Johnston, 2003). A este respecto y de forma precautoria, Turner et al. (2003), consideran que los disturbios que emanan de la propia sociedad también deben ser objeto de atención.

Desde la antropología social, Obrist et al,. (2010) consideran que la resiliencia social no solo debe contemplar al capital social, sino también al natural, financiero y físico. Además, mencionan que debe hacerse distinción entre los procesos de construcción (pre perturbación) y los procesos de manifestación (post perturbación) de resiliencia, sumado al hecho de que la resiliencia social debe ser estudiada a multi nivel: individual, colectivo y normativo; todo ello resulta relevante para tener una mejor comprensión de los procesos de conectividad y potencialidad del sistema, así como su interacción y retroalimentación con otras escalas.

El campo de la sociología argumenta que la adaptabilidad y conectividad no funcionan igual en los colectivos humanos que en el ámbito ecológico, ya que la esfera social tiene la capacidad de tomar decisiones basadas en un análisis de su entorno, a la vez que la cultura en general, y específicamente, la cosmovisión, etnia y las relaciones no formales, pueden influir en el actuar de un grupo ante un shock o perturbación; por consiguiente, su comportamiento no se limita al impulso natural (Davidson, 2010; Cote y Nightingale, 2012). Asimismo, la propia complejidad social señala la pertinencia de considerar y caracterizar los intereses que los agentes tienen sobre la resiliencia, así como sus esfuerzos por implantarla y controlarla, ya que alrededor de ella -tal cual ha ocurrido con la sostenibilidad- se erigen diferentes apreciaciones y discursos que se integran en la dinámica del poder no solo a nivel local, sino a multi escala (Keck y Sakdapolrak, 2013), lo cual resulta un punto a tener en cuenta en cuestión de la influencia de las instituciones en la toma de decisiones.

La psicología social, expone que existen dos niveles de resiliencia en los grupos de personas. A nivel individual, factores como la confianza, iniciativa, el reconocimiento de una identidad propia y la capacidad de autonomía, fomentan que un sujeto pueda enfrentar adversidades con mayor facilidad. A nivel comunitario, la razón por la que un grupo social puede ser resiliente o no, está supeditada al contexto sociocultural y la capacidad de agencia colectiva (Grotberg, 2006; Puig y Rubio, 2011). Sin embargo, al centrar su análisis en la psique, deja en segundo plano la inherencia existente con los elementos ecológicos. 
En contraposición a las disciplinas previamente mencionadas, los estudios sobre resiliencia social efectuados desde el enfoque de las ciencias ambientales, hacen hincapié en los aspectos físicos y biológicos (Marshall y Marshall, 2007) por ende, enfatizan en el hecho de que la resiliencia social depende en gran medida de los recursos físicos y la manera en la que se gestionan por parte de la comunidad. Sin embargo, los propios estudios reconocen que se requiere un mejor entendimiento de los procesos sociales que permitan una solución integral de las problemáticas que puedan existir en un socio-ecosistema (Maclean et al., 2017).

\subsection{El Turismo y su relación con la Resiliencia Social.}

Hall (2018) señala que los primeros estudios sobre la resiliencia social en el turismo se originaron a comienzos del presente siglo, como consecuencia de los esfuerzos por profundizar en el análisis interdisciplinario mediante la incorporación de nuevos enfoques, entre ellos, el del ciclo adaptativo de los sistemas socio-ecológicos, mismo que permitiría indagar en la forma en la que se estudian los destinos turísticos al incorporar distintas disciplinas y metodologías para la resolución holística de problemáticas complejas, así como la articulación entre la gestión del espacio turístico y la sostenibilidad (Farrell y Twining-Ward, 2004). Dicho enfoque, además proporcionaría el reconocimiento del turismo como un fenómeno no lineal y multi causal, al igual que el fomento de un análisis más certero de los impactos que genera (Cochrane, 2010; Strickland-Munro et al., 2010; Luthe y Wyss, 2014; Espiner el al,. 2017), razón por la que demanda mayor atención tanto de académicos, como de los tomadores de decisiones vinculados. Sobre todo, al considerar que la velocidad y temporalidad de los diversos impactos socio-ecológicos generados por la actividad, no transcurren en el espacio-tiempo de manera homogénea (Lew, 2014).

Cabe resaltar que, aunque exista la distinción de la dinámica social por la propia naturaleza del turismo (Higgins-Desbiolles, 2006; Butler, 2017), tampoco hay una constante en la delimitación de los atributos que conforman la resiliencia social. Si bien ésta se ha analizado y determinado in situ en comunidades relativamente pequeñas y que guardan un fuerte sentido de pertenencia (Ruiz-Ballesteros, 2011; Sheppard, 2017a, 2017b; EspesoMolinero, 2018; Palomino y López, 2019), su operacionalización en contextos con mayor diversidad demográfica (y por ende, complejidad) podría diferir; esto sumado al hecho de que debe guardar una mayor consideración de los aspectos ecológicos y su influencia en la dinámica social.

Con el afán de enfocar la discusión en el avance de la comprensión de la resiliencia social en destinos turísticos, en el siguiente apartado se exponen los elementos identificados para su estudio interdisciplinar, así como una propuesta para su análisis y modelación como parte del sistema socio-ecológico.

\section{CUARTA FASE: FORMULACIÓN Y MODELACIÓN DE LA TEORÍA}

\subsection{Resiliencia Social y Turismo: limitantes y coyunturas teórico-prácticas.}

Las diversas perspectivas sobre resiliencia, ponen de manifiesto la reflexión crítica de diversos postulados. En primera instancia, la resiliencia social, así como sus componentes, han sido definidos de distintas formas en función de la disciplina (o las disciplinas) que han intentado abordarla, razón por la cual, adolece de un marco teórico concreto y hay disparidad en ciertos elementos que la conforman (Puig y Rubio, 2011). Del mismo modo, su observación y operacionalización se ha visto afectada debido a que es un constructo científico que no 
puede ser observado ni medido de manera directa, sino que es inferido a través del comportamiento y las manifestaciones sociales (Obrist et al., 2010).

En cuanto al turismo, si bien existen esfuerzos académicos por abordar su resiliencia, se ha priorizado el enfoque de los subsistemas turísticos, lo cual confirma desde la revisión de la literatura conducida, que la resiliencia social se desarrolla -o al menos se ha estudiado hasta el momento- en el contexto de la centralización de los elementos inmersos en el turismo a nivel local (Jiménez, 2005). Hall (2018) sugiere que la ambigüedad teórica ha provocado que su ejecución por parte de los tomadores de decisiones en la mayoría de los contextos turísticos, se concentre en una reinterpretación de la resiliencia ingenieril, entendiéndose como un proceso cuyo fin es volver a la normalidad tras sufrir un shock (generalmente de origen natural), derivado principalmente del interés por mantener los índices de competitividad y agilizar la recuperación económica de la actividad turística tras situaciones de desastre (Basurto et al., 2020). Esto deja de lado la dinámica e interacción de otros actores y elementos que pueden influir en la gestión de un sistema socio-ecológico a mediano y largo plazo, sobre todo al considerar que la resiliencia no implica necesariamente "volver hacia atrás" sino que incluso puede incentivar su transformación si se cuentan con las capacidades adaptativas pertinentes (Rodríguez et al,. 2014; Ruiz-Ballesteros, 2017).

Ahora bien, es necesario reconocer las aportaciones y coincidencias en el estudio de la resiliencia social. Evidentemente, los enfoques concuerdan en que hay heterogeneidad y una no linealidad en los sistemas sociales, que existe una dinámica panárquica entre sistemas; $y$ que, a pesar de su inherencia, es necesario reconocer las particularidades de la esfera social y ecológica debido a que hay ciertos elementos que no pueden ser abordados de la misma forma.

Adicionalmente, se muestra un claro interés desde la academia por abordar el turismo mediante el paradigma de la resiliencia, ya que busca profundizar en la comprensión de su dinámica en los socio-ecosistemas, elaborar aproximaciones para reducir la incertidumbre ante shocks y perturbaciones, así como reconocer las capacidades de adaptación y transformación de la esfera social. Por lo tanto, también se considera que el análisis de la resiliencia social, es capaz de contribuir en la mejora de la gestión sostenible de los destinos turísticos (Butler, 2017).

\subsubsection{Resiliencia Social en Destinos Turísticos. Propuesta de análisis y modelación como parte del Sistema Socio-Ecológico.}

Así, en un intento por sintetizar y marcar puntos de convergencia entre las ideas de los autores citados, se propone entender la resiliencia social como la capacidad de un individuo, grupo o comunidad (esfera social) para enfrentar, adaptarse y transformar su entorno ante los distintos shocks y perturbaciones causados por la dinámica panárquica entre socioecosistemas. A partir del concepto construido y del análisis de la información recopilada, se presenta un encuadre interdisciplinar en el que se han logrado identificar cinco elementos que son considerados relevantes por la literatura para el estudio de la resiliencia social a saber: la agencia local, el capital social, la gobernanza, la espacio-temporalidad y la glocalidad. La psicología ha centrado el análisis de las capacidades de agencia de los individuos y cómo perciben su entorno; la antropología, además de considerar la agencia local, hace énfasis en la colaboración entre grupos como una aptitud de suma importancia para lidiar con procesos de cambio. Para las ciencias ambientales, tanto el capital social, como los procesos de 
gobernanza, son primordiales para una adecuada gestión del recurso natural. La sociología además de considerar estos últimos dos elementos, señala la glocalidad como un elemento importante para el conocimiento de la influencia de las instituciones en la toma de decisiones. Finalmente, la geografía humana alude a la valoración de cuatro de los cinco elementos identificados para un análisis pertinente de la resiliencia en la esfera social (agencia local, capital social, glocalidad y espacio-temporalidad). Si bien los elementos no son abordados en forma homóloga por las cinco especialidades científicas que han escrutado en la resiliencia social, esto a su vez confirma su alcance en función de su objeto de estudio y la necesidad de avanzar en la comprensión de la resiliencia social de forma interdisciplinar y multi escala (figura 5).

Figura 5.- Encuadre interdisciplinar sobre resiliencia social.

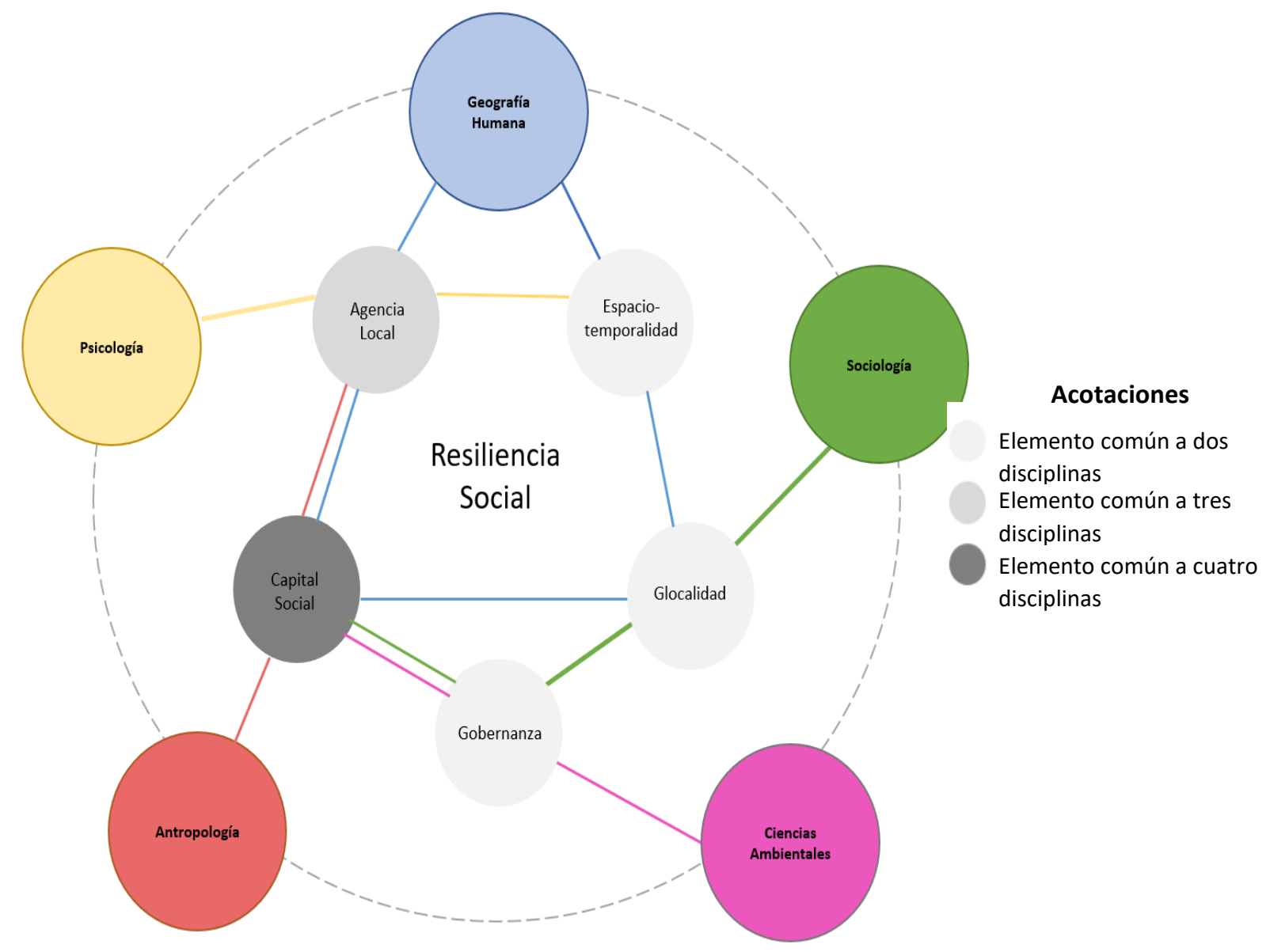

Fuente: Elaboración propia con base en los autores citados. 
Estas sinergias entre autores y disciplinas, pueden derivar en su operacionalización para poder analizar la resiliencia social a partir de su abordaje empírico en los destinos turísticos, pero desde una postura holística que podría profundizar en su complejidad (figura 6). La agencia local se define como la capacidad individual y colectiva para tomar decisiones racionales basadas en la interpretación del entorno (Adger,2000; Long, 2007; Davidson, 2010; Cochrane, 2010). Es un componente central para gestionar situaciones de cambio, ya que puede facilitar procesos de organización (tanto formales como informales) a partir de actitudes y comportamientos a favor de un bienestar tanto individual como colectivo (Matarrita-Castante et al., 2010; Espeso-Molinero, 2017; Ruiz-Ballesteros, 2017).

El capital social alude a la conexión y capacidad de colaboración entre diversos grupos que comparten recursos para alcanzar metas comunes o complementarias (Putnam, 1993; Paton y Johnston, 2003; Obrist et al., 2010; Espiner et al., 2017). En el turismo facilita la formación de redes colaborativas entre diversos grupos (Ruiz-Ballesteros, 2011; Zhao et al.,2011), lo que a su vez puede permitir el incremento de los beneficios en un destino determinado (Park et al., 2012). Su observación y análisis puede realizarse ya sea mediante métodos cualitativos (López, 2014), cuantitativos (Park et al., 2012; González, 2020) e incluso mixtos (Zhao et al., 2011).

La gobernanza refiere a la capacidad de participación multinivel de distintos actores e instituciones involucrados en la toma de decisiones en un contexto determinado (Marshall y Marshall; 2007; Keck y Sakdapolrak 2013; Maclean et al., 2014; Sheppard 2017b; Bramwell y Lane, 2011). Para fomentar una mejor organización tanto de los procesos participativos, como de la estructura de las líneas de acción para los planes de gestión local vinculados a la dinámica turística, es necesario estudiar la gobernanza de manera interdisciplinaria. Al respecto, Baggio et al. (2010) y Beaumont y Dredge (2010), consideran que su análisis debe ser efectuado desde la perspectiva de redes complejas, pues ello permite identificar las relaciones de poder e intereses de los entes involucrados en el turismo a nivel local, regional, e incluso internacional.

La espacio-temporalidad guarda relación con el conocimiento cronológico de la formación y actualidad del espacio social, así como de la influencia del entorno físico en los aspectos culturales en la escala sistémica local (Adger 2000; Cote y Nightingale, 2012; Lew, 2014). Conocer la evolución física y social es imprescindible para entender el pasado y presente de un sitio particular. Ante ello, el análisis de datos e indicadores ambientales y demográficos vinculados al bienestar y desarrollo local, medidos en lapsos consecutivos antes y después del comienzo de la actividad turística, pueden ser de gran utilidad para determinar cambios generados por el turismo en un territorio determinado (Phillimore y Goodson, 2004; Hall y Page, 2006; Sheppard, 2017a).

La glocalidad presta atención a los efectos derivados de la interacción socio-espacial multinivel (local-global) entre distintos grupos sociales (Folke, 2006; Biggs, 2012; Keck y Sakdapolrak, 2013; Greiner y Sakdapolrak, 2012). Si bien el turismo ha sido visto como una herramienta que coadyuva a alcanzar las metas globales de desarrollo sostenible (Saarinen et al., 2011), las repercusiones ecológicas y sociales derivadas de la movilidad temporal de personas tanto a nivel local como global, deben ser tomadas en cuenta para una mejor comprensión de la resiliencia en el turismo (Farrell y Twining-Ward, 2004). Al respecto, Milne y Ateljevic (2001) arguyen que se requiere "abrazar la complejidad" en los estudios turísticos para analizar su nexo global-local a mayor profundidad. En este orden de ideas, Williams 
(2009) coincide con los autores previos al sugerir que una planeación local del turismo puede ser más efectiva si se reconocen las interrelaciones socio-geográficas multi nivel de su praxis (local, regional, nacional e internacional). Ante ello, métodos interdisciplinarios que utilicen el análisis etnográfico y socio-espacial como técnicas de investigación, pueden permitir un avance en el reconocimiento de la glocalidad como factor de cambio en el turismo (Coles et al., 2005; Williams, 2009).

Figura 6. Elementos para el análisis de la resiliencia social en destinos turísticos.

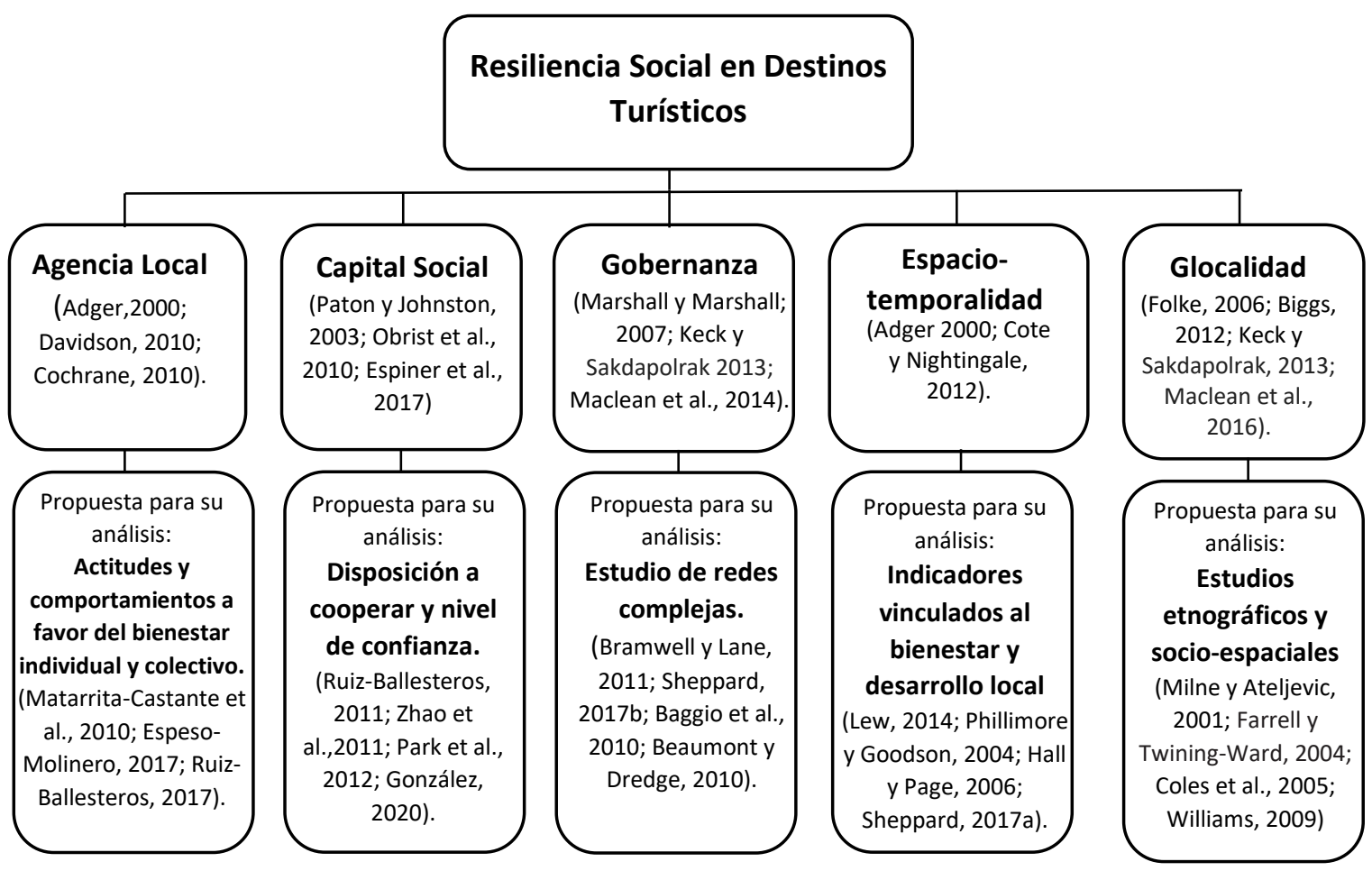

Fuente: Elaboración propia con base en los autores citados.

Es de suma importancia remarcar la interrelación de los elementos identificados, resultado de la retroalimentación multinivel en los socio-ecosistemas. Es decir, la agencia local facilita los procesos organizativos locales, lo que fomenta la disposición para cooperar entre distintos colectivos (capital social), y una toma de decisiones más equilibrada entre los involucrados (gobernanza). Todo ello permite a una interpretación análoga del entorno (espacio-temporalidad) entre actores e instituciones -locales y externos- (glocalidad), que, a largo plazo, puede conducir a una mejor gestión turística y, por ende, a la mejora en la calidad de vida en un destino determinado.

Una vez identificados los elementos que intervienen en la resiliencia social, se retoman los argumentos de Walker et al. (2012), así como las representaciones gráficas del ciclo adaptativo de Holling y Gunderson (2002) para proponer un modelo cuyo fin (sin que el proceso signifique descartar la inherencia entre dinámicas sociales y ecológicas) consiste en reconocer la existencia de las dos esferas inmersas en la dinámica de los sistemas socioecológicos (social y ecológica) que pueden (y deben) ser analizadas de acuerdo a sus especificidades en los destinos turísticos. Si bien ambas esferas cursarán las cuatro fases del ciclo adaptativo, estas tendrán fluctuaciones distintas a causa de las diferencias en la 
temporalidad de los shocks y perturbaciones que afectan en mayor medida a cada esfera mientras recorren el bucle en el que se refleja el curso de su resiliencia (figura 7).

Figura 7. Representación gráfica de la resiliencia social y ecológica de los socio-ecosistemas.

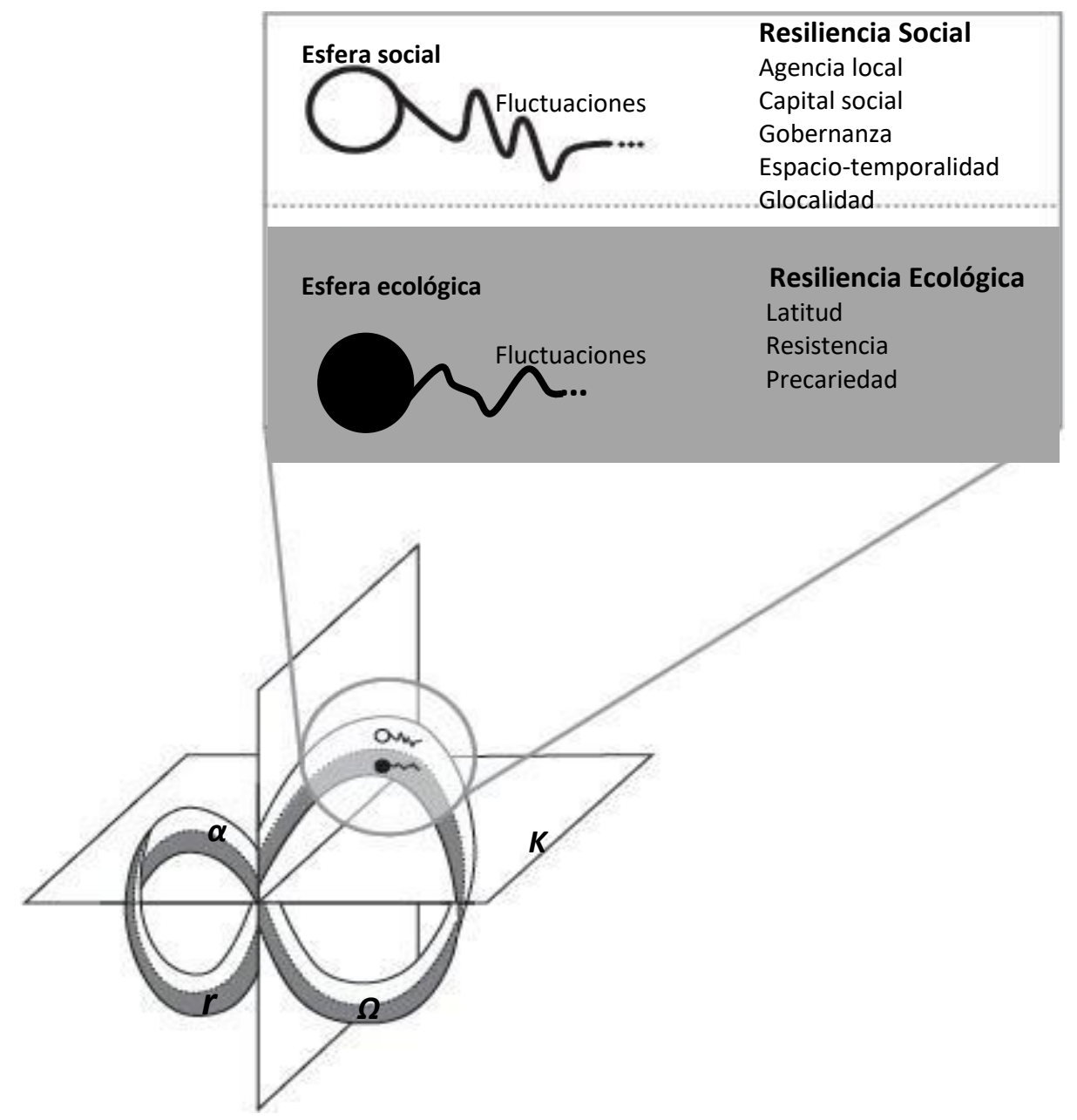

Fuente: elaboración propia con base en los autores citados.

Así, tras haber sustentado la necesidad del estudio del turismo de manera holística y descentralizada, el modelo previo se amplifica para dar paso al reconocimiento de la panarquía entre sistemas. En alusión a los modelos propuestos por Holling et al,. (2002); y Folke (2006), se comprende al turismo como uno de los muy variados fenómenos que -al estar inmerso en la interconexión y retroalimentación de los socio-ecosistemas a multi nivel- puede causar shocks y perturbaciones en una escala determinada (destino turístico) que detonarían la fase de liberación (revuelta); ante este escenario, y por la naturaleza del fenómeno, el turismo influirá directamente en la esfera social del sistema, misma que generará una respuesta en función de sus capacidades de adaptación y transformación para enfrentar situaciones de cambio (rememoria), indispensable en el ámbito de los procesos de aprendizaje (Williams y Hummelbrunner, 2010) de los actores involucrados con la actividad turística; de ahí la importancia del estudio y conocimiento de la resiliencia social en los destinos (Cochrane, 2010; Strickland-Munro t al,. 2010; Espiner et al., 2017) (figura 8). 
Figura 8. Representación del curso de la resiliencia social y ecológica en un destino

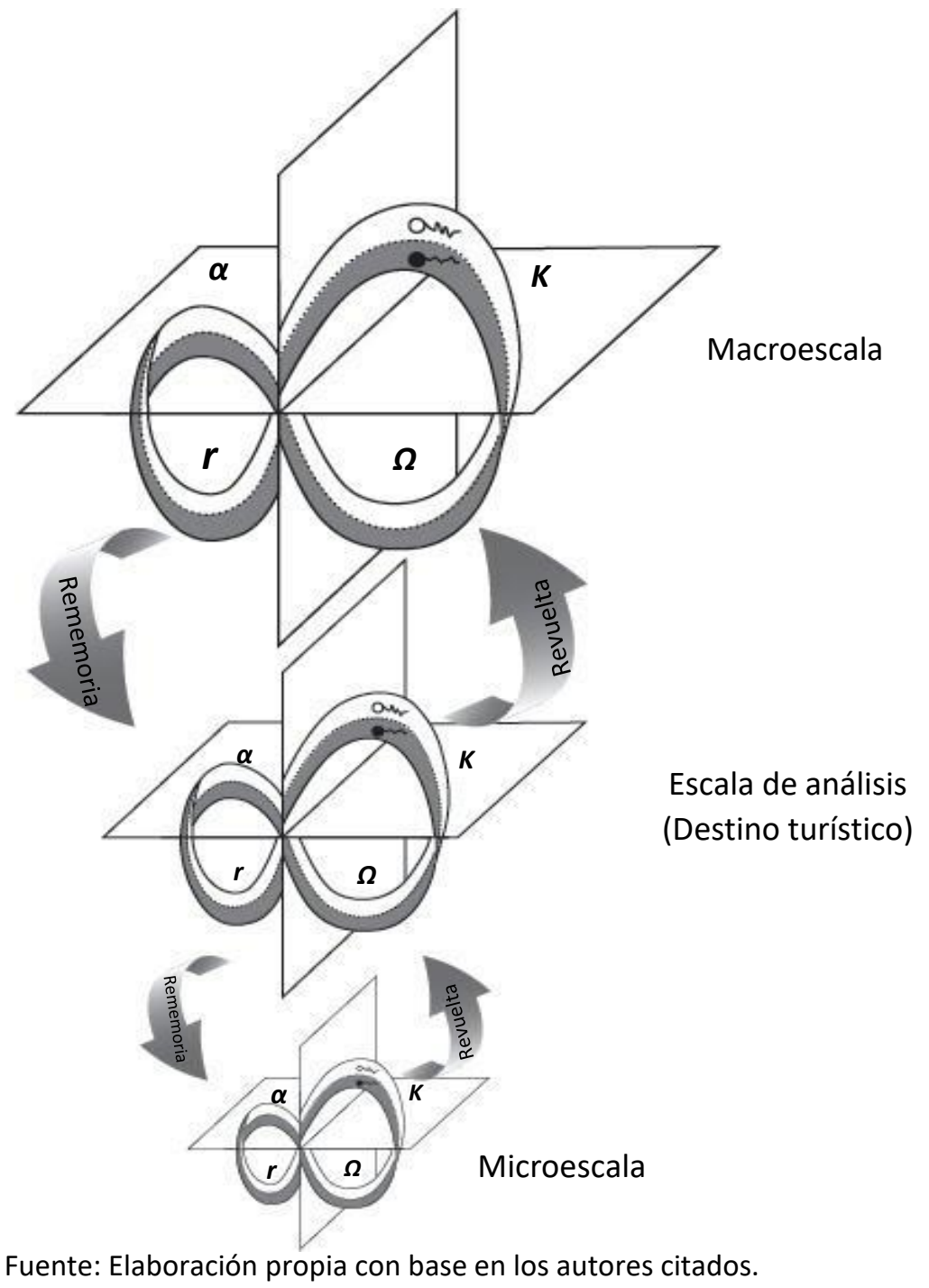

Esta visión compleja, puede conducir hacia una metodología holística que trascienda la uni y multidisciplina (Lang et al., 2012) para el estudio y solución de los diversos shocks y perturbaciones en un destino, a la vez que puede esclarecer por qué ciertos residentes locales se manifiestan a favor o en contra del turismo, por qué existe o no la articulación entre diversos grupos sociales de una comunidad, la razón por la que se facilitan o dificultan los procesos participativos y la influencia de instituciones y actores (tanto locales como externos) en la toma de decisiones. El seguimiento y propuestas de solución multi actor a las problemáticas (tanto sociales como ecológicas) detectadas, posibilitará a su vez estrategias que impulsarán o fortalecerán las capacidades necesarias para paulatinamente gestionar el turismo adecuadamente, esto debido a que la comprensión de las realidades desde la perspectiva de otros actores, puede conciliar un enfoque que permita la ejecución de acciones prácticas desde el rol y conocimiento de cada sector (Williams y Hummelbrunner, 2010). Si un destino llega a cumplir lo anteriormente expuesto, podrá catalogarse como socialmente resiliente y en ese sentido, podrá referirse al turismo (al menos para el contexto en cuestión) como una herramienta de desarrollo sostenible en un socio-ecosistema. 


\section{CONCLUSIONES}

La abstracción de los modelos sistémicos no siempre permite contemplar elementos inmersos y características de las dinámicas complejas en su totalidad, situación que se complica cuando se profundiza en la dinámica entre sistemas con características distintas. De tal manera que, al reconocer que existe un sistema socio-ecológico compuesto por dos esferas (social y ecológica) es necesario hacer distinción dos tipos de resiliencia: resiliencia ecológica y resiliencia social; razón por la que a lo largo de este trabajo se argumenta que esta última debe ser analizada desde sus especificidades para un mejor entendimiento de su dinámica interna y su vínculo con el entorno social y biofísico a multi escala.

Aunque indagar en la resiliencia social represente un reto por la dificultad que implica su observación y medición, el ejercicio de teorización propuesto, puede facilitar un discurso común para varias disciplinas, lo que a su vez puede significar un sendero para la articulación interdisciplinar para la comprensión de la esfera social en un sistema al reconocer la no linealidad, capacidad de colaboración, valores y cultura, influencia en la toma de decisiones locales y socio-espaciales a multi escala y características propias de los grupos de personas. Esto también puede facilitar el estudio de dinámicas que son de naturaleza social, tal como es el caso del turismo. En dicho tenor, es necesario realizar esfuerzos para fomentar un entendimiento del turismo como fenómeno complejo, que sea común a los diversos actores involucrados, lo cual es posible desde este enfoque.

A partir de la revisión crítica de la literatura, la identificación de vacíos en el estudio de la resiliencia social en los destinos turísticos, la argumentación de la necesidad de su estudio a partir de la consideración de los elementos a analizar desde distintas disciplinas, así como la elaboración y explicación de un modelo teórico en el que se incorpora una distinción de la esfera social y ecológica, se puede confirmar el cumplimiento en la elaboración de las cuatro fases para la teorización propuestas por Swedberg (2012). Por ende, este trabajo representa un avance en la reconceptualización teórica del turismo, ya que si bien se han desarrollado distintos modelos sistémicos que intentan describir los elementos y dinámicas del turismo, adolecen de una abstracción que permita avanzar en la comprensión de los shocks, perturbaciones, actores e influencia económica, social y ambiental del fenómeno de manera holística, razón por la que también el modelo teórico propuesto, argumenta la factibilidad de categorizar al turismo no como sistema, sino como un fenómeno inmerso en la propia dinámica y retroalimentación de los socio-ecosistemas a multi escala.

Sin embargo, la propuesta carece de ciertos alcances que invitan a la consideración de futuras líneas de investigación. Una de las principales limitaciones, es que, aunque el presente trabajo alude a la forma de analizar la resiliencia social en los destinos turísticos a partir de los elementos identificados, hasta la fecha no se han operacionalizado y analizado de manera conjunta en un contexto en concreto, por lo que resulta pertinente avanzar en la delimitación de una metodología inter (o trans) disciplinaria para la evaluación de la resiliencia social de manera empírica. Del mismo modo, se debe indagar en la relación existente entre los elementos identificados y cómo influyen significativamente en las capacidades de adaptación y transformación del socio-ecosistema para confirmar su utilidad en la resolución de los problemas complejos en un destino turístico, culminando esto en el alcance práctico de la teoría para la mejora en la gestión sostenible de los destinos turísticos. 
No obstante, se puede considerar al presente trabajo como una propuesta teórica precursora en la convergencia interdisciplinaria para la comprensión de la resiliencia social en los destinos turísticos, que permite comprender de mejor forma sus impactos y complejidad. Esto puede significar también una primera aproximación para la resolución multi-actor de los shocks y perturbaciones que el turismo genera, lo que debe ser prioridad para constatar su contribución en el ámbito de la sostenibilidad.

\section{REFERENCIAS BIBLIOGRÁFICAS:}

Adger, W. N. (2000). Social and ecological resilience: are they related? Progress in Human Geography, 24(3), 347-364. doi.org/10.1191/030913200701540465.

Allen, C. R., Angeler, D. G., Garmestani, A. S., Gunderson, L. H., y Holling, C. S. (2014). Panarchy: theory and application. Ecosystems, 17(4), 578-589. doi.org/10.1007/s10021-013-9744-2.

Araral, E. (2013). What makes socio-ecological systems robust? An institutional analysis of the 2,000 year-old Ifugao society. Human Ecology, 41(6), 859-870.

Atwool, N. (2006). Attachment and resilience: Implications for children in care. Child Care in Practice, 12(4), 315-330.

Baggio, R., Scott, N y Cooper, C. (2010). Improving tourism destination governance: a complexity science approach. Tourism Review, 65(4), 51-60.

Basurto, E. M., Pennington-Gray, L., y Basurto, X. A. (2020). Recuperación de los Destinos Turísticos Después de un Desastre: Una Evaluación del Scorecard de Resiliencia Turística para Destinos (SRTD). PASOS Revista de Turismo y Patrimonio Cultural, 18(2), 309-321.

Beaumont, N., y Dredge, D. (2010). Local tourism governance: A comparison of three network $\begin{array}{llll}\text { approaches. Journal of } & \text { Sustainable }\end{array}$ doi.org/10.1080/09669580903215139.

Bec, A., McLennan, C. L., y Moyle, B. D. (2015). Community resilience to long-term tourism decline and rejuvenation: A literature review and conceptual model. Current Issues in Tourism, 19(5), 431-457. doi.org/10.1080/13683500.2015.1083538.

Berkes, F. y Folke, C. (2002). Back to the Future: Ecosystem Dynamics and Local Knowledge. En Gunderson, L., y Holling, C. Panarchy: Understanding Transformations in Human and Natural Systems. (pp. 121-146). London: Island Press.

Biggs, R., Schlüter, M., Biggs, D., Bohensky, E. L., BurnSilver, S., Cundill, G., Dakos, V., Daw, T., Evans, L., Kotschy, K., Leitch, A., Meek, C., Quinlan, A., Raudsepp-Hearne, C., Robards, M., Schoon, M., Schultz, L y West, P. C. (2012). Toward principles for enhancing the resilience of ecosystem services. Annual Review of Environment and Resources, 37, 421-448. doi.org/10.1146/annurev-environ-051211-123836.

Bowlby, J. (1951). Menthal Care and Menthal Health. Geneva: WHO.

Bowlby, J. (1969). Attachment and Loss: I. Attachment. London: Hogarth Press. 
Bramwell, B., y Lane, B. (2011). Critical research on the governance of tourism and sustainability. Journal of Sustainable Tourism, 19(4-5), 411-421. doi.org/10.1080/09669582.2011.580586.

Buckle, P. (2006). Assessing Social Resilience. En Paton, D., \& Johnston, D. (Coords). Disaster resilience: an integrated approach. (pp. 88-104). Springfield: Charles C Thomas Publisher.

Butler, R. (2017). Tourism and Resilience. UK: CABI.

Cochrane, J. (2010). The sphere of tourism resilience. Tourism Recreation Research, 35(2), 173-185. doi.org/10.1080/02508281.2010.11081632.

Coles, T., Duval, D. T. y Hall, M. C. (2005). Tourism, mobility and global communities: New approaches to theorizing tourism and tourist spaces. En Theobald, W. (Coord.) Global Tourism. (463-481). UK: Routledge.

Cote, M., y Nightingale, A. J. (2012). Resilience thinking meets social theory: situating social change in socio-ecological systems (SES) research. Progress in Human Geography, 36(4), 475-489. doi.org/10.1177/0309132511425708.

Davidson, D. J. (2010). The applicability of the concept of resilience to social systems: some sources of optimism and nagging doubts. Society and Natural Resources, 23(12), 11351149. doi.org/10.1080/08941921003652940.

Davidson, T. (2005). What are travel and tourism: are they really an industry? En Theobald, W. (Coord.) Global Tourism. (pp. 25-31). UK: Routledge.

Espeso-Molinero, P. (2018). Collaborative capacity building as a resilience strategy for tourism development in indigenous Mexico. En Cheer, M. y Lew, A. (Eds). Tourism, Resilience and Sustainability, Adapting to Social, Political and Economic Change. (pp. 184-201). London: Routledge.

Espiner, S., Orchiston, C., y Higham, J. (2017). Resilience and sustainability: ¿A complementary relationship? Towards a practical conceptual model for the sustainability-resilience nexus in tourism. Journal of Sustainable Tourism, 25(10), 1385-1400. doi.org/10.1080/09669582.2017.1281929.

Farrell, B. H., y Twining-Ward, L. (2004). Reconceptualizing tourism. Annals of Tourism Research, 31(2), 274-295. doi.org/10.1016/i.annals.2003.12.002

Fielding, J. H. (1937). Impact resilience in testing channel black. Industrial \& Engineering Chemistry, 29(8), 880-885.

Folke, C. (2006). Resilience: The emergence of a perspective for social-ecological systems analyses. Global Environmental Change, 16(3), 253-267. doi.org/10.1016/j.gloenvcha.2006.04.002.

Gershenson, C. (2013). ¿Cómo hablar de complejidad? Llengua, Societat i Comunicació, 11, 14-19.

González, A. (2020). Capital social y calidad de vida de los habitantes de un destino turístico de sol y playa en el Caribe Mexicano. En Bringas, N., Osorio, M. y Sosa, A. (Coords.) Casos de Planeación y Gestión Turística: comportamientos, problemas y avances. (pp. 223-242). México: AMIT. 
González-Quintero, C., y Avila-Foucat, V. S. (2019). Operationalization and Measurement of Social-Ecological Resilience: A Systematic Review. Sustainability, 11(21), 6073. doi.org/10.3390/su11216073

Greiner, C., y Sakdapolrak, P. (2012). Rural-urban migration, agrarian change, and the environment in Kenya: a critical review of the literatura. Population and environment, 34(4), 524-553.

Grotberg, E. H. (2006). ¿Qué entendemos por resiliencia? En Grotberg, E. (Ed). La resiliencia en el mundo de hoy: cómo superar las adversidades. (pp.17-58). Barcelona: Gedisa Editorial.

Hall, C. y Page, S. (2006). The Geography of Tourism and Recreation, Environment, Place and Space. London: Routledge.

Hall, M. (2018). Resilience in tourism, Development, Theory, and application. En Cheer, M. y Lew, A. (Eds). Tourism, Resilience and Sustainability, Adapting to Social, Political and Economic Change. (18-33). London: Routledge.

Higgins-Desbiolles, F. (2006). More than an "industry": The forgotten power of tourism as a social force. Tourism management, 27(6), 1192-1208.

Hodgson, D., McDonald, J. L., y Hosken, D. J. (2015). What do you mean, 'resilient'? Trends in ecology \& evolution, 30(9), 503-506.

Holling, C. (1973). Resilience and stability of ecological systems. Annual Review of Ecology and Systematics, 4(1), 1-23.

Holling, C., y Gunderson, L. (2002). Resilience and adaptive cycles. En Gunderson, L., y Holling, C. Panarchy: Understanding Transformations in Human and Natural Systems. (pp. 2562). London: Island Press.

Holling, C., Gunderson, L. y Peterson, G. (2002). Sustainability and Panarchies. En Gunderson, L., y Holling, C. Panarchy: Understanding Transformations in Human and Natural Systems. (pp. 63-102). London: Island Press.

Jafari, J. (2005). El turismo como disciplina científica. Política y sociedad, 42(1), 39-56.

Jafari, J., y Ritchie, J. B. (1981). Toward a framework for tourism education: Problems and prospects. Annals of Tourism Tesearch, 8(1), 13-34.

Jiménez, A. (2005). Una aproximación a la conceptualización del turismo desde la teoría general de sistemas. México: Universidad del Caribe.

Kates, R. W., Clark, W., Corell, R., Hall, J., Jaeger, C., Lowe, I., McCarty, J., Schellnhuber, H., Bolin,B., Dickson, N., Faucheux, S., Gallopin, G., Grübler, A., Huntley, B., Jägger, J., Jodha,N., Kasperson, R., Mabogunje, A., Matson, P., Mooney, H., Moore III, B., O’Riordan, T. y Svedin, U. (2001). Sustainability science. Science, 292(5517), 641-642.

Keck, M., y Sakdapolrak, P. (2013). What is social resilience? Lessons learned and ways forward. Erdkunde, 5-19.

Lang, D. J., Wiek, A., Bergmann, M., Stauffacher, M., Martens, P., Moll, P., ... \& Thomas, C. J. (2012). Transdisciplinary research in sustainability science: practice, principles, and challenges. Sustainability science, 7(1), 25-43. doi.org/10.1007/s11625-011-0149-x. 
Leiper, N. (1979). The framework of tourism: Towards a definition of tourism, tourist, and the tourist industry. Annals of Tourism Research, 6(4), 390-407.

Lew, A. (2014). Scale, change and resilience in community tourism planning. Tourism Geographies, 16(1), 14-22. doi.org/10.1080/14616688.2013.864325.

Long, N. (2007). Sociología del desarrollo: una perspectiva centrada en el actor. México: CIESAS.

López, V. (2014). El capital social en las empresas indígenas de turismo. Su análisis y dinamización en la red expediciones Sierra Norte, México (Tesis doctoral, Universitat de Girona).

Luthe, T., y Wyss, R. (2014). Assessing and planning resilience in tourism. Tourism Management, 44, 161-163. doi.org/10.1016/j.tourman.2014.03.011.

Maclean, K., Cuthill, M., y Ross, H. (2014). Six attributes of social resilience. Journal of Environmental Planning and Management, 57(1), 144-156. doi.org/10.1080/09640568.2013.763774.

Maclean, K., Ross, H., Cuthill, M., y Witt, B. (2017). Converging disciplinary understandings of social aspects of resilience. Journal of Environmental Planning and Management, 60(3), 519-537.

Marshall, N. A., y Marshall, P. A. (2007). Conceptualizing and operationalizing social resilience within commercial fisheries in northern Australia. Ecology and Society, 12(1). Recuperado de http://www.ecologyandsociety.org/vol12/iss1/art1/.

Mason, P. (2008). Tourism: Impacts, planning and management. Oxford, UK: ButterworthHeinemann.

Matarrita-Cascante, D., Brennan, M. A., y Luloff, A. E. (2010). Community agency and sustainable tourism development: The case of La Fortuna, Costa Rica. Journal of Sustainable Tourism, 18(6), 735-756. doi.org/10.1080/09669581003653526.

Milne, S. y Ateljevic, I. (2001). Tourism, economic development and the global-local nexus: Theory embracing complexity. Tourism Geographies: An International Journal of Tourism Space, Place and Environment, 3(4), 369-393. doi.org/10.1080/146166800110070478.

Moustakas, C. (1990). Heuristic research: Design, methodology, and applications. California, USA: Sage Publications.

Obrist, B., Pfeiffer, C., y Henley, R. (2010). Multi-layered social resilience: A new approach in mitigation research. Progress in Development Studies, 10(4), 283-293. doi.org/10.1177/146499340901000402.

OMT (2005). Indicadores de desarrollo sostenible para los destinos turísticos. España: Organización Mundial del Turismo.

Ostrom, E. (2009). A general framework for analyzing sustainability of social-ecological systems. Science, 325(5939), 419-422. DOI: 10.1126/science.1172133. 
Palomino, B., y López, G. (2019). Relaciones del turismo de naturaleza, la comunalidad y la resiliencia en la Sierra Norte de Oaxaca, México. PASOS, Revista de Tursimo y Patrimonio Cultural. 17(6), 1205-12016. doi .org/10.25145/ j .pasos.2019.17.083.

Park, D. B., Lee, K. W., Choi, H. S., \& Yoon, Y. (2012). Factors influencing social capital in rural tourism communities in South Korea. Tourism Management, 33(6), 1511-1520.

Paton, D., y Johnston, D. (2006). Disaster resilience: an integrated approach. Springfield: Charles C Thomas Publisher.

Phillimore, J. y Goodson, L. (Eds.). (2004). Qualitative research in tourism: Ontologies, epistemologies and methodologies. New York: Routledge.

Puig, G., y Rubio, J. (2011). Manual de resiliencia aplicada. Madrid: Editorial Gedisa.

Putnam, R. (1993). The prosperous community: Social capital and public life. The american prospect, 13. Disponible en: http://www. prospect. org/print/vol/13.

Rodríguez, I., Williams, A. M., y Hall, C. M. (2014). Tourism innovation policy: Implementation and outcomes. Annals of Tourism Research, 49, 76-93. doi.org/10.1016/j.annals.2014.08.004.

Ruiz-Ballesteros, E. (2011). Social-ecological resilience and community-based tourism: an approach from Agua Blanca, Ecuador. Tourism Management, 32(3), 655-666. doi.org/10.1016/i.tourman.2010.05.021.

Ruiz-Ballesteros, E. (2017). Socio-ecological balance in Community-based Tourism Experiences: a Research Proposal. En Butler, R. (Ed). Tourism and Resilience. (pp. 4150). UK: CABI.

Rutter, M. (1971). Parent-child separation: Psychological effects on the children. Journal of child psychology and psychiatry, 12(4), 233-260.

Rutter, M. (1985). Resilience in the face of adversity: Protective factors and resistance to psychiatric disorder. The British Journal of Psychiatry, 147(6), 598-611.

Saarinen, J., Rogerson, C., y Manwa, H. (2011). Tourism and Millennium Development Goals: tourism for global development? Current Issues in Tourism. 14(3),201-203. doi.org/10.1080/13683500.2011.555180.

Scoville, M. (1942). Wartime tasks of pshychiatric social workers in Great Britain. American Journal of Psychiatry, 99(3), 358-363.

Sheppard, V. (2017a). Resilience and Destinaition Governance. En Butler, R. (Ed). Tourism and Resilience. (pp. 53-68). UK: CABI.

Sheppard, V. (2017b). Resilience and Destination Governance: Whistler, B.C. En Butler, R. (Ed.) Tourism and Resilience. (pp. 69-80). UK: CABI.

Strickland-Munro, J. K., Allison, H. E., y Moore, S. A. (2010). Using resilience concepts to investigate the impacts of protected area tourism on communities. Annals of Tourism Research, 37(2), 499-519. doi.org/10.1016/i.annals.2009.11.001. 
Swedberg, R. (2012). Theorizing in sociology and social science: Turning to the context of discovery. Theory and society, 41(1), 1-40.

Tejeida, R., Cruz, E y Briones, A. (2016). Sistémica y Turismo. México: Miguel Ángel Porrúa.

Theobald, W. (1998). Global tourism. UK: Routledge.

Turner, B., Matson, P., McCarthy, J., Corell, R., Christensen, L., Eckley, N. \& Tyler, N. (2003). Illustrating the coupled human-environment system for vulnerability analysis: Three case studies. Proceedings of the National Academy of Sciences, 100(14), 80808085.

Turner, L. y Ash, J. (1976). The golden hordes: International tourism and the pleasure periphery. New York: St. Martin's Press.

Von Bertalanffy, L. (1969). General systems theory and psychiatry, an overview. En Gray, W., Duhl, F. y Rizzo, N. (Coords). General Systems Theory and Psychiatry. (pp. 33-46). Boston: Little Brown and Company.

Von Bertalanffy, L. (1976). Teoría General de los Sistemas. México: Fondo de Cultura Económica.

Wagner, P. (1999). The twenieth century, the century of the Social Sciences? En Kazancigil, A., y Makinson, D. (Eds.).World social science report 1999. (pp. 16-31). Paris: Unesco.

Walker, B., Holling, C., Carpenter, S. y Kinzig, A. (2004). Resilience, adaptability and transformability in social-ecological systems. Ecology and Society, 9(2), Disponible en: http://www.ecologyandsociety.org/vol9/iss2/art5. Consultado el: 03/01/2019.

Williams, B. and Hummelbrunner, R. (2010) Systems Concepts in Action: A Practitioner's Toolkit. Stanford: Stanford University Press

Williams, S. (2009). Tourism Geography, a new synthesis. London: Routledge.

Zhao, W., Ritchie, J. B., y Echtner, C. M. (2011). Social capital and tourism entrepreneurship. Annals of Tourism Research, 38(4), 1570-1593.

Zhu, J., y Ruth, M. (2013). Exploring the resilience of industrial ecosystems. Journal of environmental management, 122, 65-75.

\section{CONTRIBUCIONES DE LOS AUTORES:}

Autor 1: Concepción original del trabajo, análisis e interpretación de los datos y redacción del contenido.

Autor 2: Interpretación de los datos, redacción y revisión crítica del contenido, aprobación para versión a publicar.

Autor 3: Revisión crítica del contenido, aprobación para versión a publicar. 


\section{AGRADECIMIENTOS:}

El primer autor, extiende su gratitud al Consejo Nacional de Ciencia y Tecnología (CONACYT) por el apoyo financiero otorgado para su proyecto doctoral, adscrito al Posgrado en Ciencias de la Sostenibilidad de la Universidad Nacional Autónoma de México; mismo que ha permitido la elaboración de este artículo. Asimismo, agradece el apoyo de la Lic. Sandra L. Guevara en la elaboración de las figuras 2 y 3 , y la reestructuración del diseño de las figuras 7 y 8 , utilizadas para la explicación de modelo teórico del presente documento. 